\title{
Hiperplasia nodular linfoide asociada con colitis ulcerativa: descripción de un caso y revisión de la literatura
}

\section{Lymphoid Nodular Hyperplasia Associated with Ulcerative Colitis: Case Study and Literature Review}

Inés Benedetti P., MD ${ }^{1}$, Julián Hoyos C. ${ }^{2}$, Rafael Carmona V. MD. ${ }^{3}$

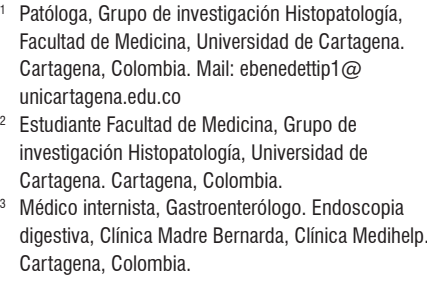

Patóloga, Grupo de investigación Histopatología, Facultad de Medicina, Universidad de Cartagena. Cartagena, Colombia. Mail: ebenedettip1@ unicartagena.edu.co

2 Estudiante Facultad de Medicina, Grupo de investigación Histopatología, Universidad de Cartagena. Cartagena, Colombia.

3 Médico internista, Gastroenterólogo. Endoscopia digestiva, Clínica Madre Bernarda, Clínica Medihelp. Cartagena, Colombia.

Fecha recibido: $18-11-15$ Fecha aceptado: 25-07-16

\begin{abstract}
Resumen
La hiperplasia nodular linfoide es una patología del aparato gastrointestinal, poco frecuente en adultos. Se caracteriza por un crecimiento del tejido linfoide mucoso y submucoso como respuesta a diferentes tipos de estímulos nocivos. Se ha descrito asociada con otras patologías como infección por VIH/ Síndrome de Inmunodeficiencia Adquirida, giardiasis, infección por Helicobacter Pylori, enfermedad celíaca, y en pocos casos con enfermedad inflamatoria intestinal. No tiene una presentación clínica específica y su manejo aún no está claro.
\end{abstract}

\section{Palabras clave}

Colitis ulcerativa, hiperplasia nodular linfoide, endoscopia gastrointestinal.

\begin{abstract}
Lymphoid nodular hyperplasia is a pathology of the gastrointestinal tract that rarely occurs among adults. It is characterized by growth of submucosal lymphoid tissue and mucosal response to different types of noxious stimuli. It has been described in association with other diseases such as HIVIAIDS, giardiasis, Helicobacter pylori infections, celiac disease, and very rarely with inflammatory bowel disease. At present, there is no specific clinical presentation or treatment.
\end{abstract}

\author{
Keywords
Ulcerative Colitis, lymphoid nodular hyperplasia, gastrointestinal endoscopy. \\ Keywords
Ulcerative Colitis, lymphoid nodular hyperplasia, gastrointestinal endoscopy.
}


sexo masculino con HNL, como hallazgo endoscópico en el contexto de una colitis ulcerativa.

\section{DESCRIPCIÓN DEL CASO}

Paciente masculino de 33 años de edad, cuadro clínico de un mes de evolución consistente en deposiciones diarreicosanguinolentas, asociadas con pujo y tenesmo rectal, y pérdida de peso. En el momento del examen físico el paciente está en buen estado general; tiene el abdomen blando, no doloroso, sin masas ni visceromegalias. Se reporta examen coprológico y hemograma normales. La esofagogastroduodenoscopia evidencia gastritis eritematosa antrocorporal, con test de ureasa negativo; se hace impresión diagnóstica de hemorragia de vías digestivas inferiores secundaria a enfermedad hemorroidal versus neoplasia colorrectal. Se realiza colonoscopia total evidenciando proctitis ulcerada con lesiones compatibles con colitis ulcerativa a $15 \mathrm{~cm}$ de la línea dentada, y múltiples lesiones nodulares polipoides de aspecto benigno distribuidas en la mucosa a nivel de íleon distal (figura 1); se toman biopsias de colon e íleon distal. El estudio anatomopatológico revela fragmentos de mucosa colónica, algunos revestidos por epitelio columnar, con criptas tubulares de patrón mucoide conservado, rodeadas por lámina propia con moderado infiltrado inflamatorio crónico mediado por linfocitos y plasmocitos. En otros fragmentos se observan criptas irregulares sin mucina, con núcleos agrandados y aumento del número de figuras mitóticas, rodeadas por lámina propia con abundantes neutrófilos y formación de microabscesos crípticos. En la lámina propia del íleon distal se observan múltiples folículos linfoides secundarios, con mantos bien definidos y centros germinales activos (figura 2). Se hace diagnóstico final de hiperplasia de las placas de Peyer-hiperplasia nodular linfoide, asociada a hallazgos de enfermedad inflamatoria intestinal, sugestivos de colitis ulcerativa.

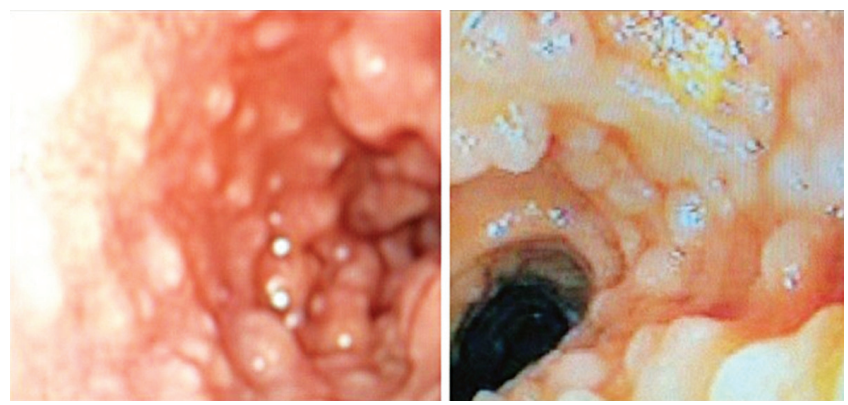

Figura 1. Aspecto endoscópico de la mucosa del íleon distal con múltiples lesiones nodulares, polipoides, de 2 a $10 \mathrm{~mm}$ de diámetro.

\section{DISCUSIÓN}

La HNL presenta una forma difusa, y una focal que compromete principalmente íleon terminal, pudiendo encontrarse también en estómago, colon y recto (2). La localización más frecuente es el intestino delgado. Aunque su patogenia no es bien conocida se propone que en personas inmunocomprometidas podría representar un mecanismo compensatorio a la pobre respuesta inmunológica en el tracto digestivo (7), mientras que en inmunocompetentes podría ser secundaria a sobrestimulación del tejido linfoide asociado con el tubo digestivo por estímulos nocivos a la mucosa, como es el caso de la EII, en donde se ha propuesto que aparece como consecuencia de una regulación a la alta del sistema inmune asociada con trastornos inmunológicos (7). Para algunos autores se considera un estadio transicional y un factor de riesgo para la aparición de linfomas intestinales y extraintestinales, aunque estos últimos son poco frecuentes (8-10). También se ha sugerido asociación entre la HNL y neoplasias colónicas no exofíticas, adenomas y adenocarcinomas (10).

Ha sido asociada con varias enfermedades como la inmunodeficiencia común variable, la deficiencia selectiva de IgA, la infección por VIH/SIDA, la infección por Helicobacter pylori, y giardiasis (11), y en los niños también se ha descrito como relacionada con alergias alimentarias (2.7). Además, la HNL suele ser asintomática, o presentar síntomas de afectación del tracto gastrointestinal que no siguen un patrón específico, ocasionando desde dolor abdominal leve, hasta obstrucción intestinal completa o sangrado abundante (12). Es así como en el reporte de una cohorte de 283 niños se describió como síntoma más frecuente la diarrea, seguida por la pérdida de peso y dolor abdominal (1).

La HNL puede detectarse por endoscopia, estudios baritados de contraste y otros como la enterorresonancia magnética $(2,14)$. Los hallazgos endoscópicos clásicos incluyen lesiones nodulares con halo eritematoso o máculas rojizas generalmente menores de $5 \mathrm{~mm}$ de diámetro; sin embargo, por la semejanza macroscópica de las lesiones con otras entidades, como los síndromes polipósicos, para su diagnóstico es necesario tomar biopsia de las lesiones y realizar estudio histopatológico. En los casos que comprometen intestino delgado los estudios baritados y la videocápsula endoscópica (VCE) son de suma utilidad para excluir complicaciones y determinar extensión de la enfermedad (13); sin embargo, no es indicación formal para diagnóstico ni seguimiento con VCE. Hay que agregar que la HNL puede ser indistinguible de la Enfermedad de Crohn en imágenes de resonancia magnética (14). 

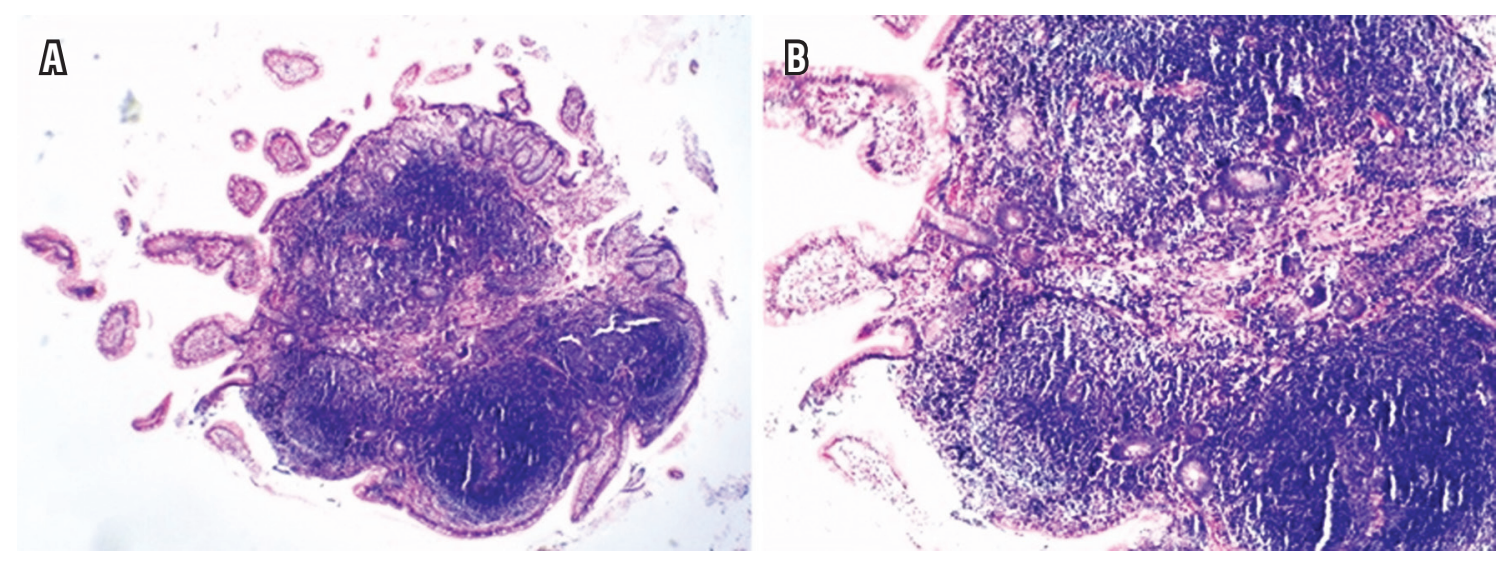

Figura 2. Fragmento de íleon con folículos linfoides hiperplásicos, centros germinales activos y mantos bien definidos, localizados en la mucosa y parte superficial de la submucosa, HE, -a:40X, -b:100X.

El diagnóstico definitivo se establece mediante el estudio histopatológico al encontrar folículos linfoides hiperplásicos con centros germinales mitóticamente activos, y mantos linfocitarios bien delimitados, inmersos en la mucosa y submucosa (2). La naturaleza polimorfa del infiltrado y la ausencia de atipia citológica, en presencia de folículos linfoides de aspecto reactivo, permiten diferenciar la hiperplasia nodular linfoide de un linfoma; en algunos casos para facilitar esta distinción se requiere el uso de inmunomarcadores y estudios moleculares (15).

El tratamiento generalmente va dirigido a la condición asociada. La erradicación de la giardiasis suele acompañarse de la resolución de los síntomas; sin embargo, la mayoría de reportes de casos no describen regresión de las lesiones (16). Algunos autores sugieren vigilancia periódica del paciente con videocápsula endoscópica por el riesgo de transformación maligna, y tomar biopsia de las lesiones de rápido crecimiento para descartar transformación linfomatosa (17).

\section{CONCLUSIONES}

La HNL es una condición poco frecuente en adultos; presenta diversas manifestaciones clínicas; su etiología se ha relacionado con trastornos del sistema inmune. Existen pocos casos reportados de asociación con EII; en este contexto podría representar una sobrerregulación de los mecanismos de respuesta del tejido linfoide asociado con el tubo digestivo. Por su aspecto endoscópico, el diagnóstico diferencial requiere realización de estudio histopatológico.

\section{REFERENCIAS}

1. Rubio-Tapia A, Hernández-Calleros J, Trinidad-Hernández S, Uscanga L. Clinical characteristics of a group of adults with nodular lymphoid hyperplasia: a single center experience. World journal of gastroenterology:WJG. 2006;12(12):1945-8.

2. Albuquerque A. Nodular lymphoid hyperplasia in the gastrointestinal tract in adult patients: A review. World J Gastrointest Endosc. 2014;6(11):534-40.

3. Colarian J, Calzada R, Jaszewski R. Nodular lymphoid hyperplasia of the colon in adults: is it common? Gastrointestinal endoscopy. 1990;36(4):421-2.

4. Ward EM, Wolfsen HC. Review article: the non-inherited gastrointestinal polyposis syndromes. Alimentary pharmacology \& therapeutics. 2002;16(3):333-42.

5. Kokkonen J, Karttunen TJ. Lymphonodular hyperplasia on the mucosa of the lower gastrointestinal tract in children: an indication of enhanced immune response? Journal of pediatric gastroenterology and nutrition. 2002;34(1):42-6.

6. Iacono G, Ravelli A, Di Prima L, Scalici C, Bolognini S, Chiappa $S$, et al. Colonic lymphoid nodular hyperplasia in children: relationship to food hypersensitivity. Clinical gastroenterology and hepatology: the official clinical practice journal of the American Gastroenterological Association. 2007;5(3):361-6.

7. Lin $\mathrm{C} \mathrm{SH}$, Kuo $\mathrm{C}$ et al. Nodular lymphoid hiperplasia in endoscopic and FDG-PET/CT. Kaohsiung J MED SCI. 2013;29(7):396-9.

8. Monsanto P, Lerias C, Almeida N, Lopes S, Cabral JE, Figueiredo $\mathrm{P}$, et al. Intestinal nodular lymphoid hyperplasia and extraintestinal lymphoma-a rare association. Acta gastroenterológica, Bélgica. 2012;75(2):260-2.

9. Chiaramonte C GS. Nodular lymphoid hyperplasia of the small bowel complicated by jejunal lymphoma in a patient with common variable immune deficiency syndrome. Am J roentgenol. 1994;163(5):1118-9.

10. CA R. Nonprotruding colorectal neoplasms: epidemiologic viewpoint. World J Surg. 2000;24(9):1098-103.

11. Khuroo MS, Khuroo NS, Khuroo MS. Diffuse duodenal nodular lymphoid hyperplasia: a large cohort of patients etiologically related to Helicobacter pylori infection. BMC Gastroenterology. 2011;11:36. 
12. Garg V, Lipka S, Rizvon K, Singh J, Rashid S, Mustacchia P. Diffuse nodular lymphoid hyperplasia of intestine in selective IgG 2 subclass deficiency, autoimmune thyroiditis, and autoimmune hemolytic anemia: case report and literature review. Journal of gastrointestinal and liver diseases. JGLD. 2012;21(4):431-4.

13. Said-Criado I, Gil-Aguado A. Nodular lymphoid hyperplasia in common variable immunodeficiency. The Lancet. 2014;383(9911):e2.

14. Plumb AA, Pendse DA, Mc Cartney S, Punwani S, Halligan S, Taylor SA. Lymphoid nodular hyperplasia of the terminal ileum can mimic active Crohn disease on MR enterography. AJR American journal of roentgenology. 2014;203(4):W400-7.
15. Tomita S, Kojima M, Imura J, Ueda Y, Koitabashi A, Suzuki $\mathrm{Y}$, et al. Diffuse nodular lymphoid hyperplasia of the large bowel without hypogammaglobulinemia or malabsorption syndrome: a case report and literature review. Int J surg pathol. 2002;10(4):297-302.

16. Luzi G, Zullo A, Iebba F, Rinaldi V, Sanchez Mete L, Muscaritoli $\mathrm{M}$, et al. Duodenal pathology and clinicalimmunological implications in common variable immunodeficiency patients. The american journal of gastroenterology. 2003;98(1):118-21.

17. Postgate A, Despott E, Talbot I, Phillips R, Aylwin A, Fraser C. An unusual cause of diarrhea: diffuse intestinal nodular lymphoid hyperplasia in association with selective immunoglobulin A deficiency (with video). Gastrointestinal endoscopy. 2009; 70(1):168-9. 